Citation: Çerdik Yaslan, H., Girgin, A., "SITEM for the Conformable Space-Time Fractional Coupled KD Equations". Journal of Engineering Technology and Applied Sciences 3 (3) 2018 : 223-233.

\title{
SITEM FOR THE CONFORMABLE SPACE-TIME FRACTIONAL COUPLED KD EQUATIONS
}

\author{
Handan Çerdik Yaslan a*, Ayşe Girgin ${ }^{a}$, \\ ${ }^{a}$ Department of Mathematics, Faculty of Science and Arts, Pamukkale University, Turkey \\ hcerdik@pau.edu.tr (" corresponding author)
}

\begin{abstract}
In the present paper, new analytical solutions for the space-time fractional coupled KonopelchenkoDubrovsky (KD) equations are obtained by using the simplified $\tan \left(\frac{\phi(\xi)}{2}\right)$ - expansion method (SITEM). Here, fractional derivatives are described in conformable sense. The obtained traveling wave solutions are expressed by the trigonometric, hyperbolic, exponential and rational functions. Simulation of the obtained solutions are given at the end of the paper.
\end{abstract}

Keywords: Space-time fractional coupled KD equations, simplified $\tan \left(\frac{\phi(\xi)}{2}\right)$-expansion method (SITEM), conformable derivative.

\section{Introduction}

In recent years, to model and describe phenomena in various fields of science such as plasma physics, nonlinear optics, nonlinear transmission lines, solid state physics, chemical kinematics, and biology, nonlinear partial differential equations have been used. The popularity of these equations is because of their capacity to model many real systems. Therefore, nonlinear equations have gained a very significant place in the current research. To solve the nonlinear partial differential equations, various methods have been developed (see, for example, $[1,2,3,4,5,6])$.

KD equations were introduced by Konopelchenko and Dubrovsky [7]. These equations constitute applications in the ocean dynamics, fluid mechanics and plasma physics. To solve the coupled KD equations, various methods have been proposed such as the standard truncated Painlevé analysis, homotopy perturbation method, generalized F-expansion method, $\left(\mathrm{G}^{\prime} / \mathrm{G}, 1 / \mathrm{G}\right)$-expansion method, first integral method, extended Riccati equation rational 
method, Xu's stable-range method, tanh-sech method, cosh-sinh method and exponential functions method $[8,9,10,11,12,13,14,15]$. There is not much work on the fractional coupled KD equation. Fractional coupled KD equations have been solved by using sub equation method, Jacobi elliptic equation method and extended $\mathrm{G}^{\prime} / \mathrm{G}$-expansion method in $[16,17,18]$. Here fractional derivatives are described in modified Riemann-Liouville sense and Caputo sense.

Recently, $\tan \left(\frac{\phi(\xi)}{2}\right)$-expansion method has been applied by many authors [19, 20, 21, 22, 23]. In [19], ITEM has been simplified and called simplified ITEM (SITEM). SITEM has been applied to Kundu-Eckhaus equation. To our knowledge, there is no other application of the SITEM in the literature. In this paper, we consider space-time fractional coupled KD equation. Here fractional derivatives are described in conformable sense. We obtain some traveling wave solutions such as trigonometric, hyperbolic, exponential and rational functions.

\section{Description of the conformable fractional derivative and its properties}

For a function $\mathrm{f}:(0, \infty) \rightarrow \mathrm{R}$, the conformable fractional derivative of $\mathrm{f}$ of order $0<\alpha<1$ is defined as (see, for example, [24])

$\mathrm{T}_{\mathrm{t}}^{\alpha} \mathrm{f}(\mathrm{t})=\lim _{\varepsilon \rightarrow 0} \frac{\mathrm{f}\left(\mathrm{t}+\varepsilon \mathrm{t}^{1-\alpha}\right)-\mathrm{f}(\mathrm{t})}{\varepsilon}$.

Some important properties of the the conformable fractional derivative are as follows:

$\mathrm{T}_{\mathrm{t}}^{\alpha}(\mathrm{af}+\mathrm{bg})(\mathrm{t})=\mathrm{aT} \mathrm{T}_{\mathrm{t}}^{\alpha} \mathrm{f}(\mathrm{t})+\mathrm{b} \mathrm{T}_{\mathrm{t}}^{\alpha} \mathrm{g}(\mathrm{t})$, for all $\mathrm{a}, \mathrm{b} \in \mathrm{R}$, $\mathrm{T}_{\mathrm{t}}^{\alpha}\left(\mathrm{t}^{\mu}\right)=\mu \mathrm{t}^{\mu-\alpha}$,

(2)

$T_{t}^{\alpha}\left(f(g(t))=t^{1-\alpha} g^{\prime}(t) f^{\prime}(g(t))\right.$.

\section{Description of the simplified $\tan \left(\frac{\phi(\xi)}{2}\right)$-expansion method (SITEM) for solving conformable partial differential equations}

Let us consider general nonlinear fractional partial differential equation of the type

$\mathrm{P}\left(\mathrm{u}, \mathrm{T}_{\mathrm{t}}^{\alpha} \mathrm{u}, \mathrm{T}_{\mathrm{x}}^{\beta} \mathrm{u}, \mathrm{T}_{\mathrm{t}}^{\alpha} \mathrm{T}_{\mathrm{t}}^{\alpha} \mathrm{u}, \mathrm{T}_{\mathrm{t}}^{\alpha} \mathrm{T}_{\mathrm{x}}^{\beta} \mathrm{u}, \mathrm{T}_{\mathrm{x}}^{\beta} \mathrm{T}_{\mathrm{x}}^{\beta} \mathrm{u}, \ldots\right)=0,0<\alpha \leq 1,0<\beta \leq 1$,

where $\mathrm{u}$ is an unknown function and $\mathrm{P}$ is a polynomial of $\mathrm{u}$ and its partial fractional derivatives. Using the following transformation

$\mathrm{u}(\mathrm{x}, \mathrm{t})=\mathrm{U}(\xi), \xi=\mathrm{k} \frac{\mathrm{t}^{\alpha}}{\alpha}+\mathrm{m} \frac{\mathrm{x}^{\beta}}{\beta}$,

where $\mathrm{k}$ and $\mathrm{m}$ are non zero arbitrary constants, Eq. (3) can be written as the following nonlinear ordinary differential equations

$\phi\left(\mathrm{U}^{\prime} \mathrm{U}^{\prime}, \mathrm{U}^{\prime \prime}, \mathrm{U}^{\prime \prime \prime}, \ldots\right)=0$.

Suppose that traveling wave solution of Eq. (5) can be expressed as follows 
$\mathrm{U}(\xi)=\sum_{\mathrm{k}=0}^{\mathrm{m}} \mathrm{A}_{\mathrm{k}}\left[\mathrm{p}+\tan \left(\frac{\phi(\xi)}{2}\right)\right]^{\mathrm{k}}+\sum_{\mathrm{k}=1}^{\mathrm{m}} \mathrm{B}_{\mathrm{k}}\left[\mathrm{p}+\tan \left(\frac{\phi(\xi)}{2}\right)\right]^{-\mathrm{k}}$,

$\phi(\xi)$ satisfies the following ordinary differential equation

$\phi^{\prime}(\xi)=\mathrm{a} \sin (\phi(\xi))+\mathrm{b} \cos (\phi(\xi))+\mathrm{c}$,

(7)

where $\mathrm{a}, \mathrm{b}, \mathrm{c}, \mathrm{A}_{\mathrm{k}}(0 \leq \mathrm{k} \leq \mathrm{m})$ and $\mathrm{B}_{\mathrm{k}}(1 \leq \mathrm{k} \leq \mathrm{m})$ are constants to be determined. The solution of Eq. (7) is given as:

For $\mathrm{b}=\mathrm{c}, \mathrm{a}=0$,

$$
\tan \left(\frac{\phi}{2}\right)=b \xi+c_{1}-p
$$

For $\mathrm{b}=\mathrm{c}, \mathrm{a} \neq 0$,

$$
\tan \left(\frac{\phi}{2}\right)=\mathrm{c}_{1} \exp (\mathrm{a} \xi)-\frac{\mathrm{b}}{\mathrm{a}} \text {. }
$$

For $\mathrm{b} \neq \mathrm{c}, \Delta=\mathrm{a}^{2}+\mathrm{b}^{2}-\mathrm{c}^{2}>0$,

$$
\tan \left(\frac{\phi}{2}\right)=\frac{2}{\mathrm{~b}-\mathrm{c}} \frac{\mathrm{c}_{1} \mathrm{r}_{1} \exp \left(\mathrm{r}_{1} \xi\right)+\mathrm{c}_{2} \mathrm{r}_{2} \exp \left(\mathrm{r}_{2} \xi\right)}{\mathrm{c}_{1} \exp \left(\mathrm{r}_{1} \xi\right)+\mathrm{c}_{2} \exp \left(\mathrm{r}_{2} \xi\right)}-\mathrm{p}
$$

For $\mathrm{b} \neq \mathrm{c}, \Delta=\mathrm{a}^{2}+\mathrm{b}^{2}-\mathrm{c}^{2}=0$,

$$
\tan \left(\frac{\phi}{2}\right)=\frac{\mathrm{a}}{\mathrm{b}-\mathrm{c}}+\frac{2}{\mathrm{~b}-\mathrm{c}} \frac{\mathrm{c}_{2}}{\mathrm{c}_{1}+\mathrm{c}_{2} \xi} \text {. }
$$

For $\mathrm{b} \neq \mathrm{c}, \Delta=\mathrm{a}^{2}+\mathrm{b}^{2}-\mathrm{c}^{2}<0$,

$$
\tan \left(\frac{\phi}{2}\right)=\frac{\mathrm{a}}{\mathrm{b}-\mathrm{c}}+\frac{\sqrt{-\Delta}}{\mathrm{b}-\mathrm{c}} \frac{-\mathrm{c}_{1} \sin \left(\frac{\sqrt{-\Delta}}{2} \xi\right)+\mathrm{c}_{2} \cos \left(\frac{\sqrt{-\Delta}}{2} \xi\right)}{\mathrm{c}_{1} \cos \left(\frac{\sqrt{-\Delta}}{2} \xi\right)+\mathrm{c}_{2} \sin \left(\frac{\sqrt{-\Delta}}{2} \xi\right)}
$$

where $c_{1}$ and $c_{2}$ are arbitrary constants, $r_{1}=(a+p(b-c)+\sqrt{\Delta}) / 2$ and $\mathrm{r}_{2}=(\mathrm{a}+\mathrm{p}(\mathrm{b}-\mathrm{c})-\sqrt{\Delta}) / 2$. Substituting Eq. (6) into Eq. (5) and by balancing the highest order derivatives and nonlinear terms appearing in Eq. (5), the value of $\mathrm{m}$ can be computed. Collecting the coefficients of $\left(p+\tan \left(\frac{\phi}{2}\right)\right)^{\mathrm{k}},\left(\mathrm{p}+\tan \left(\frac{\phi}{2}\right)\right)^{-\mathrm{k}}(\mathrm{k}=0,1,2, \ldots)$, we have system of algebraic equations. Solving the system with the aid of the Mathematica, the values of $\mathrm{A}_{0}$, $\mathrm{A}_{\mathrm{k}}, \mathrm{B}_{\mathrm{k}}(\mathrm{k}=1,2, \ldots, \mathrm{m}), \mathrm{a}, \mathrm{b}, \mathrm{c}$ and $\mathrm{p}$ are computed.

\section{Application}

Conformable space-time fractional Konopelchenko-Dubrovsky equation is given in the following from [18]

$$
\begin{aligned}
& \mathrm{T}_{\mathrm{t}}^{\alpha} \mathrm{u}+\mathrm{T}_{\mathrm{x}}^{\beta} \mathrm{T}_{\mathrm{x}}^{\beta} \mathrm{T}_{\mathrm{x}}^{\beta} \mathrm{u}-6 \lambda_{2} \mathrm{uT}_{\mathrm{x}}^{\beta} \mathrm{u}+\frac{3}{2} \lambda_{1}^{2} \mathrm{u}^{2} \mathrm{~T}_{\mathrm{x}}^{\beta} \mathrm{u}-3 \mathrm{~T}_{\mathrm{y}}^{\theta} \mathrm{v}+3 \lambda_{1} \mathrm{vT}_{\mathrm{x}}^{\beta} \mathrm{u}=0, \\
& \mathrm{~T}_{\mathrm{y}}^{\theta} \mathrm{u}=\mathrm{T}_{\mathrm{x}}^{\beta} \mathrm{v}, 0<\alpha \leq 1,0<\beta \leq 1 .
\end{aligned}
$$

Let us consider the following transformation 
$\mathrm{u}(\mathrm{x}, \mathrm{y}, \mathrm{t})=\mathrm{U}(\xi), \mathrm{v}(\mathrm{x}, \mathrm{y}, \mathrm{t})=\mathrm{V}(\xi), \xi=\mathrm{k} \frac{\mathrm{t}^{\alpha}}{\alpha}+\mathrm{m} \frac{\mathrm{x}^{\beta}}{\beta}+\mathrm{n} \frac{\mathrm{y}^{\theta}}{\theta}$,

where k, m, nare constants. Substituting (15) into Eqs.(13)-(14), we obtain the following differential equations

$\mathrm{kU}^{\prime}-\mathrm{m}^{3} \mathrm{U}^{\prime \prime \prime}-6 \lambda_{2} \mathrm{mUU} \mathrm{U}^{\prime}+\frac{3}{2} \lambda_{1}^{2} \mathrm{mU}^{2} \mathrm{U}^{\prime}-3 n \mathrm{~V}^{\prime}+3 \lambda_{1} \mathrm{mU^{ \prime }} \mathrm{V}=0$,

$\mathrm{n} \mathrm{U}^{\prime}=\mathrm{m} \mathrm{V}^{\prime}$.

Integrating of Eqs.(16)-(17) with zero constant of integration and eliminating V, we have

$$
\left(\mathrm{k}-\frac{3 \mathrm{n}^{2}}{\mathrm{~m}}\right) \mathrm{U}+\left(3 \lambda_{1} \mathrm{n}-6 \lambda_{2} \mathrm{~m}\right) \frac{\mathrm{U}^{2}}{2}+\frac{\lambda_{1}^{2} \mathrm{~m}}{2} \mathrm{U}^{3}-\mathrm{m}^{3} \mathrm{U}^{\prime \prime}=0 .
$$

Let us suppose that the solution of Eq.(18) can be expressed in the form Eq.(6) for $p=0$. Substituting Eq.(6) into Eq.(18) and then by balancing the highest order derivative term and nonlinear term in result equation, the value of $\mathrm{m}$ can be determined as 1 . Therefore, (6) reduces to

$$
\mathrm{U}(\xi)=\mathrm{A}_{0}+\mathrm{A}_{1}\left[\tan \left(\frac{\phi(\xi)}{2}\right)\right]+\mathrm{B}_{1}\left[\tan \left(\frac{\phi(\xi)}{2}\right)\right]^{-1} .
$$

Substituting Eq.(19) into (18), collecting all the terms with the same power of $\tan \left(\frac{\phi}{2}\right)$,

we can obtain a set of algebraic equations for the unknowns $A_{0}, A_{1}, B_{1}, k, m, n$ :

$$
\begin{gathered}
\mathrm{A}_{1}^{3} \lambda_{1}^{2} \mathrm{~m}^{2}-\mathrm{A}_{1} \mathrm{~b}^{2} \mathrm{~m}^{4}+2 \mathrm{~A}_{1} \mathrm{bcm}^{4}-\mathrm{A}_{1} \mathrm{c}^{2} \mathrm{~m}^{4}=0, \\
3 \mathrm{~A}_{0} \mathrm{~A}_{1}^{2} \lambda_{1}^{2} \mathrm{~m}^{2}-6 \mathrm{~A}_{1}^{2} \lambda_{2} \mathrm{~m}^{2}+3 \mathrm{a}_{1} \mathrm{bm}^{4}-3 \mathrm{a}_{1} \mathrm{~cm}^{4}+3 \mathrm{~A}_{1}^{2} \lambda_{1} \mathrm{mn}=0, \\
-2 \mathrm{a}^{2} \mathrm{~A}_{1} \mathrm{~m}^{4}+3 \mathrm{~A}_{0}^{2} \mathrm{~A}_{1} \lambda_{1}^{2} \mathrm{~m}^{2}+6 \mathrm{~A}_{0} \mathrm{~A}_{1} \lambda_{1} \mathrm{mn}-12 \lambda_{2} \mathrm{~A}_{0} \mathrm{~A}_{1} \mathrm{~m}^{2}+3 \mathrm{~B}_{1} \mathrm{~A}_{1}^{2} \lambda_{1}^{2} \mathrm{~m}^{2} \\
+\mathrm{A}_{1} \mathrm{~b}^{2} \mathrm{~m}^{4}-\mathrm{A}_{1} \mathrm{c}^{2} \mathrm{~m}^{4}+2 \mathrm{kA}_{1} \mathrm{~m}-6 \mathrm{~A}_{1} \mathrm{n}^{2}=0, \\
2 \mathrm{~A}_{0} \mathrm{~km}-6 \mathrm{~A}_{0}^{2} \lambda_{2} \mathrm{~m}^{2}-6 \mathrm{~A}_{0} \mathrm{n}^{2}+\mathrm{A}_{0}^{3} \lambda_{1}^{2} \mathrm{~m}^{2}-\mathrm{a} \mathrm{A}_{1} \mathrm{bm}^{4}-\mathrm{aA}_{1} \mathrm{~cm}^{4}+a b \mathrm{~B}_{1} \mathrm{~m}^{4} \\
-\mathrm{aB}_{1} \mathrm{~cm}^{4}-12 \mathrm{~A}_{1} \mathrm{~B}_{1} \lambda_{2} \mathrm{~m}^{2}+3 \mathrm{~A}_{0}^{2} \lambda_{1} \mathrm{mn}+6 \mathrm{~A}_{0} \mathrm{~A}_{1} \mathrm{~B}_{1} \lambda_{1}^{2} \mathrm{~m}^{2}+6 \mathrm{~A}_{1} \mathrm{~B}_{1} \lambda_{1} \mathrm{mn}=0, \\
-2 \mathrm{a}^{2} \mathrm{~B}_{1} \mathrm{~m}^{4}+3 \mathrm{~A}_{0}^{2} \mathrm{~B}_{1} \lambda_{1}^{2} \mathrm{~m}^{2}+6 \mathrm{~A}_{0} \mathrm{~B}_{1} \lambda_{1} \mathrm{mn}-12 \lambda_{2} \mathrm{~A}_{0} \mathrm{~B}_{1} \mathrm{~m}^{2}+\mathrm{b}^{2} \mathrm{~B}_{1} \mathrm{~m}^{4} \\
+3 \mathrm{~A}_{1} \mathrm{~B}_{1}^{2} \lambda_{1}^{2} \mathrm{~m}^{2}-\mathrm{B}_{1} \mathrm{c}^{2} \mathrm{~m}^{4}+2 \mathrm{kB}_{1} \mathrm{~m}-6 \mathrm{~B}_{1} \mathrm{n}^{2}=0, \\
3 \mathrm{~A}_{0} \mathrm{~B}_{1}^{2} \lambda_{1}^{2} \mathrm{~m}^{2}-6 \mathrm{~B}_{1}^{2} \lambda_{2} \mathrm{~m}^{2}-3 \mathrm{abB}_{1} \mathrm{~m}^{4}-3 \mathrm{aB}_{1} \mathrm{~cm}^{4}+3 \mathrm{~B}_{1}^{2} \lambda_{1} \mathrm{mn}=0, \\
-\mathrm{b}^{2} \mathrm{~B}_{1} \mathrm{~m}^{4}-2 \mathrm{bB}_{1} \mathrm{~cm}^{4}+\mathrm{B}_{1}^{3} \lambda_{1}^{2} \mathrm{~m}^{2}-\mathrm{B}_{1} \mathrm{c}^{2} \mathrm{~m}^{4}=0 .
\end{gathered}
$$

Solving the algebraic equations in the Mathematica, we obtain the following set of solutions:

Case 1: $A_{0}= \pm \frac{a n}{2 \lambda_{2}}, \quad A_{1}=\mp \frac{(b-c) n}{2 \lambda_{2}}, \quad B_{1}=0, \quad m=\frac{\lambda_{1} n}{2 \lambda_{2}}$, $\mathrm{k}=\frac{96 \lambda_{2}^{4} \mathrm{n}-\left(\mathrm{a}^{2}+\mathrm{b}^{2}-\mathrm{c}^{2}\right) \lambda_{1}^{4} \mathrm{n}^{3}}{16 \lambda_{1} \lambda_{2}^{3}}$

For $\mathrm{b}=\mathrm{c}$ and $\mathrm{a} \neq 0$,

$$
\mathrm{U}_{1,2}(\xi)= \pm \frac{\mathrm{an}}{2 \lambda_{2}} \text {. }
$$

For $\Delta>0$ and $\mathrm{b} \neq \mathrm{c}$, 
$\mathrm{U}_{3,4}(\xi)= \pm \frac{\mathrm{an}}{2 \lambda_{2}} \mp \frac{\mathrm{n}}{\lambda_{2}}\left[\frac{\mathrm{c}_{1} \frac{\mathrm{a}+\sqrt{\Delta}}{2} \exp \left(\frac{\mathrm{a}+\sqrt{\Delta}}{2} \xi\right)+\mathrm{c}_{2} \frac{\mathrm{a}-\sqrt{\Delta}}{2} \exp \left(\frac{\mathrm{a}-\sqrt{\Delta}}{2} \xi\right)}{\mathrm{c}_{1} \exp \left(\frac{\mathrm{a}+\sqrt{\Delta}}{2} \xi\right)+\mathrm{c}_{2} \exp \left(\frac{\mathrm{a}-\sqrt{\Delta}}{2} \xi\right)}\right]$.

For $\Delta=0$ and $\mathrm{b} \neq \mathrm{c}$,

$\mathrm{U}_{5,6}(\xi)= \pm \frac{\mathrm{an}}{2 \lambda_{2}} \mp \frac{\mathrm{n}}{2 \lambda_{2}}\left[\mathrm{a}+\frac{2 \mathrm{c}_{2}}{\mathrm{c}_{1}+\mathrm{c}_{2} \xi}\right]$.

For $\Delta<0$ and $\mathrm{b} \neq \mathrm{c}$,

$\mathrm{U}_{7,8}(\xi)= \pm \frac{\mathrm{an}}{2 \lambda_{2}} \mp \frac{\mathrm{n}}{2 \lambda_{2}}\left[\mathrm{a}+\sqrt{-\Delta} \frac{-\mathrm{c}_{1} \sin \left(\frac{\sqrt{-\Delta}}{2} \xi\right)+\mathrm{c}_{2} \cos \left(\frac{\sqrt{-\Delta}}{2} \xi\right)}{\mathrm{c}_{1} \cos \left(\frac{\sqrt{-\Delta}}{2} \xi\right)+\mathrm{c}_{2} \sin \left(\frac{\sqrt{-\Delta}}{2} \xi\right)}\right]$.

Case 2: $A_{0}= \pm \frac{\text { an }}{2 \lambda_{2}}, \quad A_{0}= \pm \frac{\text { an }}{2 \lambda_{2}} \quad A_{-} 1=0, \quad B_{1}= \pm \frac{(b+c) n}{2 \lambda_{2}}, \quad m=\frac{\lambda_{1} n}{2 \lambda_{2}}$, $\mathrm{k}=\frac{96 \lambda_{2}^{4} \mathrm{n}-\left(\mathrm{a}^{2}+\mathrm{b}^{2}-\mathrm{c}^{2}\right) \lambda_{1}^{4} \mathrm{n}^{3}}{16 \lambda_{1} \lambda_{2}^{3}}$

For $\mathrm{b}=\mathrm{c}$ and $\mathrm{a}=0$,

$\mathrm{U}_{9,10}(\xi)= \pm \frac{\mathrm{bn}}{\lambda_{2}}\left[\mathrm{~b} \xi+\mathrm{c}_{1}\right]^{-1}$.

For $\mathrm{b}=\mathrm{c}$ and $\mathrm{a} \neq 0$,

$\mathrm{U}_{11,12}(\xi)= \pm \frac{\mathrm{an}}{2 \lambda_{2}} \pm \frac{\mathrm{bn}}{\lambda_{2}}\left[\mathrm{c}_{1} \exp (\mathrm{a} \xi)-\frac{\mathrm{b}}{\mathrm{a}}\right]^{-1}$.

For $\Delta>0$ and $\mathrm{b} \neq \mathrm{c}$,

$\mathrm{U}_{13,14}(\xi)= \pm \frac{\mathrm{an}}{2 \lambda_{2}} \pm \frac{(\mathrm{b}+\mathrm{c}) \mathrm{n}}{4 \lambda_{2}}\left[\frac{1}{\mathrm{~b}-\mathrm{c}} \frac{\mathrm{c}_{1} \frac{\mathrm{a}+\sqrt{\Delta}}{2} \exp \left(\frac{\mathrm{a}+\sqrt{\Delta}}{2} \xi\right)+\mathrm{c}_{2} \frac{\mathrm{a}-\sqrt{\Delta}}{2} \exp \left(\frac{\mathrm{a}-\sqrt{\Delta}}{2} \xi\right)}{\mathrm{c}_{1} \exp \left(\frac{\mathrm{a}+\sqrt{\Delta}}{2} \xi\right)+\mathrm{c}_{2} \exp \left(\frac{\mathrm{a}-\sqrt{\Delta}}{2} \xi\right)}\right]$.

For $\Delta=0$ and $\mathrm{b} \neq \mathrm{c}$,

$$
\mathrm{U}_{15,16}(\xi)= \pm \frac{\mathrm{an}}{2 \lambda_{2}} \pm \frac{(\mathrm{b}+\mathrm{c}) \mathrm{n}}{2 \lambda_{2}}\left[\frac{\mathrm{a}}{\mathrm{b}-\mathrm{c}}+\frac{2}{\mathrm{~b}-\mathrm{c}} \frac{\mathrm{c}_{2}}{\mathrm{c}_{1}+\mathrm{c}_{2} \xi}\right]^{-1} \text {. }
$$

For $\Delta<0$ and b $\neq \mathrm{c}$,

$\mathrm{U}_{17,18}(\xi)= \pm \frac{\mathrm{an}}{2 \lambda_{2}} \pm \frac{(\mathrm{b}+\mathrm{c}) \mathrm{n}}{2 \lambda_{2}}\left[\frac{\mathrm{a}}{\mathrm{b}-\mathrm{c}}+\frac{\sqrt{-\Delta}}{\mathrm{b}-\mathrm{c}} \frac{-\mathrm{c}_{1} \sin \left(\frac{\sqrt{-\Delta}}{2} \xi\right)+\mathrm{c}_{2} \cos \left(\frac{\sqrt{-\Delta}}{2} \xi\right)}{\mathrm{c}_{1} \cos \left(\frac{\sqrt{-\Delta}}{2} \xi\right)+\mathrm{c}_{2} \sin \left(\frac{\sqrt{-\Delta}}{2} \xi\right)}\right]^{-1}$.

Here $\xi=\left(\frac{96 \lambda_{2}^{4} \mathrm{n}-\left(\mathrm{a}^{2}+\mathrm{b}^{2}-\mathrm{c}^{2}\right) \lambda_{1}^{4} \mathrm{n}^{3}}{16 \lambda_{1} \lambda_{2}^{3}} \frac{\mathrm{t}^{\alpha}}{\alpha}+\frac{\lambda_{1} \mathrm{n}}{2 \lambda_{2}} \frac{\mathrm{x}^{\beta}}{\beta}+\mathrm{n} \frac{\mathrm{x}^{\theta}}{\theta}\right)$.

Figs. 1 and 2 show 3D and 2D plots of the king wave solution $\mathrm{u}_{4}(\mathrm{x}, 0.25, \mathrm{t})$ and $\mathrm{u}_{4}(\mathrm{x}, 0.25,1) \quad$ in $(21)$ for $\alpha=0.75, \beta=1, \theta=0.5 \quad, \quad \lambda_{1}=0.25, \lambda_{2}=0.2 \quad, \quad \mathrm{n}=1$, $\mathrm{a}=3, \mathrm{~b}=2, \mathrm{c}=1, \mathrm{c}_{1}=2, \mathrm{c}_{2}=1$, respectively. 
Figs. 3 and 4 show 3D and 2D plots of the periodic wave solution $\mathrm{u}_{8}(\mathrm{x}, 1, \mathrm{t})$ and $\mathrm{u}_{8}(\mathrm{x}, 1,1) \quad$ in Eq. (23) for $\alpha=0.5, \beta=1, \theta=0.5 \quad, \quad \lambda_{1}=0.25, \lambda_{2}=0.2 \quad, \quad \mathrm{n}=-1$, $\mathrm{a}=0.1, \mathrm{~b}=0.2, \mathrm{c}=0.5, \mathrm{c}_{1}=2, \mathrm{c}_{2}=1$, respectively.

Figs.5 and 6 show 3D and 2D plots of the solitary wave solution $\mathrm{a}=0.1, \mathrm{~b}=0.2, \mathrm{c}=0.5, \mathrm{c}_{1}=2, \mathrm{c}_{2}=1 \quad \mathrm{u}_{12}(\mathrm{x}, 1, \mathrm{t}) \quad$ and $\quad \mathrm{u}_{12}(\mathrm{x}, 1,1) \quad$ in Eq. (25) for $\alpha=0.5, \beta=1, \theta=0.5, \quad \lambda_{1}=0.25, \lambda_{2}=0.2 \quad, \quad \mathrm{n}=-2 \quad, \quad \mathrm{a}=3, \mathrm{~b}=1, \mathrm{c}=1, \mathrm{c}_{1}=1, \mathrm{c}_{2}=2 \quad$, respectively.

Figs.7 and 8 show 3D and 2D plots of the periodic wave solution $\mathrm{u}_{18}(\mathrm{x}, 1, \mathrm{t})$ and $\mathrm{u}_{18}(\mathrm{x}, 1,1) \quad$ in Eq.(28) for $\alpha=0.5, \beta=1, \theta=0.5 \quad, \quad \lambda_{1}=0.25, \lambda_{2}=0.2 \quad, \quad \mathrm{n}=-2 \quad$, $\mathrm{a}=0.1, \mathrm{~b}=0.2, \mathrm{c}=0.5, \mathrm{c}_{1}=2, \mathrm{c}_{2}=1$, respectively.

\section{Conclusion}

In this paper, the conformable space-time fractional coupled KD equations have been solved by using the simplified $\tan \left(\frac{\phi(\xi)}{2}\right)$-expansion method (SITEM) and new exact traveling wave solutions containing hyperbolic, trigonometric, exponential and rational functions have been obtained. Note that SITEM has been applied to the Kundu-Eckhaus equation only for the parameter $p=0$ in [19]. In the literature, fractional coupled KD equations with modified Riemann-Liouville and Caputo fractional derivatives have been investigated. In our work, SITEM has been applied to space-time fractional coupled KD equations with conformable fractional derivative.

\section{References}

[1] Dehghan, M., Manafian, J., "The Solution of the Variable Coefficients Fourth- Order Parabolic Partial Differential Equations by the Homotopy Perturbation Method.", Zeitschrift für Naturforschung A. 64. 7-8 (2009): 420-430.

[2] Dehghan, M., Manafian J., Saadatmandi, A., "Solving Nonlinear Fractional Partial Differential Equations Using the Homotopy Analysis Method." Num. Meth. Partial Differential Eq.: An International Journal 26.2 (2010): 448-479.

[3] Dehghan, M., Manafian, J., Saadatmandi, A., “Analytical treatment of some partial differential equations arising in mathematical physics by using the Exp-function method.’' Int. J. Modern Phys. B 25.22 (2011): 2965-2981.

[4] Foroutan, M., Zamanpour, I., Manafian, J., “Applications of IBSOM and ETEM for solving the nonlinear chains of atoms with long-range interactions." Eur. Phys. J. Plus 132.10 (2017): 421.

[5] Seyedi, S. H., Saray, B. N., Nobari, M. R. H., “Using interpolation scaling functions based on Galerkin method for solving non-Newtonian fluid flow between two vertical flat plates." Appl. Math. Comput. 269 (2015): 488-496.

[6] Seyedi, S. H., Saray, B. N., Ramazani, A., "'On the multiscale simulation of squeezing nanofluid flow by a highprecision scheme.' Powder Technology 340 (2018): 264-273.

[7] Konopelchenko, B. G., Dubrovsky, V. G., "Some new integrable nonlinear evolution equations in (2+1)-dimensions.'” Phys. Lett. 102 (1984): 15-17. 
[8] Ji, L., Sen-Yue, L, Ke-Lin, W., "Multi-soliton solutions of the KonopelchenkoDubrovsky equation.' Chin. Phys. Lett. 18.9 (2001): 1173.

[9] Song, L., Zhang, H., "Application of the extended homotopy perturbation method to a kind of nonlinear evolution equations.' Appl. Math. Comput. 197.1 (2008): 87-95.

[10] Zhang, S., Xia, T., "A generalized F-expansion method and new exact solutions of Konopelchenko-Dubrovsky equations.' Appl. Math. Comput. 183.2 (2006): 11901200.

[11] Yasar, E, Giresunlu, I. B., "'Exact Traveling Wave Solutions and Conservation Laws of (2+1) Dimensional Konopelchenko-Dubrovsky System.' IJNS 22.2 (2016): 118-128.

[12] Taghizadeh, N., Mirzazadeh, M., "Exact Travelling Wave Solutions for Konopelchenko-Dubrovsky Equation by the First Integral Method.' Appl. Appl. Math. 6 (2011): 153-161.

[13] Cao, B., "Solutions of Jimbo-Miwa Equation and Konopelchenko-Dubrovsky Equations.' Acta Appl Math 112.2 (2010): 181-203.

[14] Wazwaz, A., "New kinks and solitons solutions to the $(2+1)$-dimensional Konopelchenko-Dubrovsky equation." Math. Comput. Model. 45.3-4 (2007): 473479.

[15] Song, L, Zhang, H., "New exact solutions for the Konopelchenko-Dubrovsky equation using an extended Riccati equation rational expansion method and symbolic computation.' Appl. Math. Comput. 187.2 (2007): 1373-1388.

[16] Liu, Y, Yan, L., "Solutions of Fractional Konopelchenko-Dubrovsky and NizhnikNovikov-Veselov Equations Using a Generalized Fractional Subequation Method." Abstr. Appl. Anal. 2013 (2013): 1-7.

[17] Zheng, B, Qinghua, F., "The Jacobi Elliptic Equation Method for Solving Fractional Partial Differential Equations." Abstr. Appl. Anal. 2014 (2014): 1- 9.

[18] Mohyud-Din, S. T., Saba, F.,. "Extended ( $\left.\mathrm{G}^{\prime} / \mathrm{G}\right)$-Expansion Method for Konopelchenko-Dubrovsky (KD) Equation of Fractional Order.' Int. J. Appl. Comput. Math 3 (2017): 161-172.

[19] Liu, H-Z, Zhang, T., “A note on the improved $\tan (\phi(\xi) / 2)$-expansion method.' Optik 131 (2017): 273-278.

[20] Manafian, J., Foroutan, M., “'Application of $\tan (\phi(\xi) / 2)$-expansion method for the time-fractional Kuramoto-Sivashinsky equation.’” Opt. Quant. Electron. 49.8 (2017): 272.

[21] Manafian, J., Lakestani, M., “'Optical soliton solutions for the Gerdjikov-Ivanov model via $\tan (\phi / 2)$-expansion method.' Optik 127.20 (2016): 9603-9620.

[22] Manafian, J., Lakestani, M., “Application of $\tan (\phi / 2)$-expansion method for solving the Biswas-Milovic equation for Kerr law nonlinearity.'’ Optik 127.4 (2016): 20402054.

[23] Hosseini, K., et al. "Resonant optical solitons with perturbation terms andfractional temporal evolution using improved $\tan (\phi(\eta) / 2)$-expansion method and exp function approach.' Optik 158 (2018): 933-939.

[24] Khalil, R., et al. "A new definition of fractional derivative.' J. Comput. Appl. Math. 264 (2014): 65-70. 


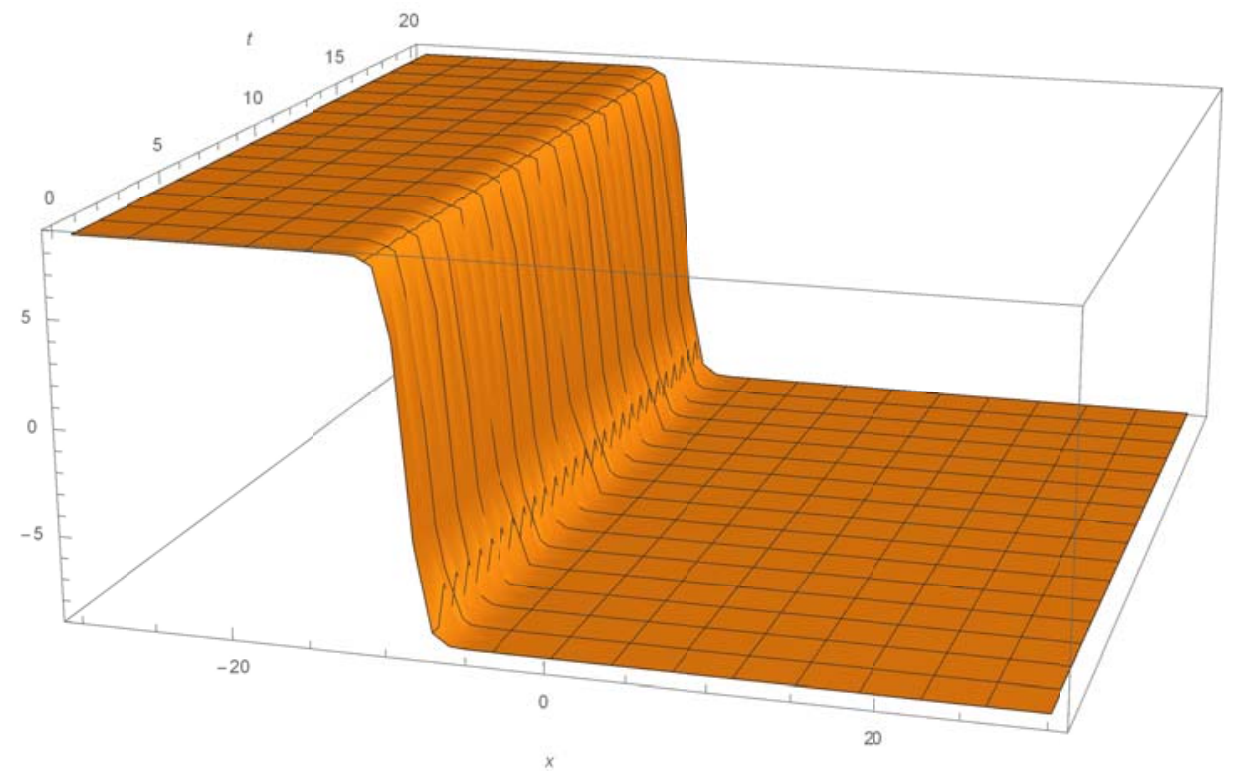

Figure 1: $3 \mathrm{D}$ plot of the obtained traveling wave solution $u_{4}(x, 0.25, t)$ of Eq.(21).

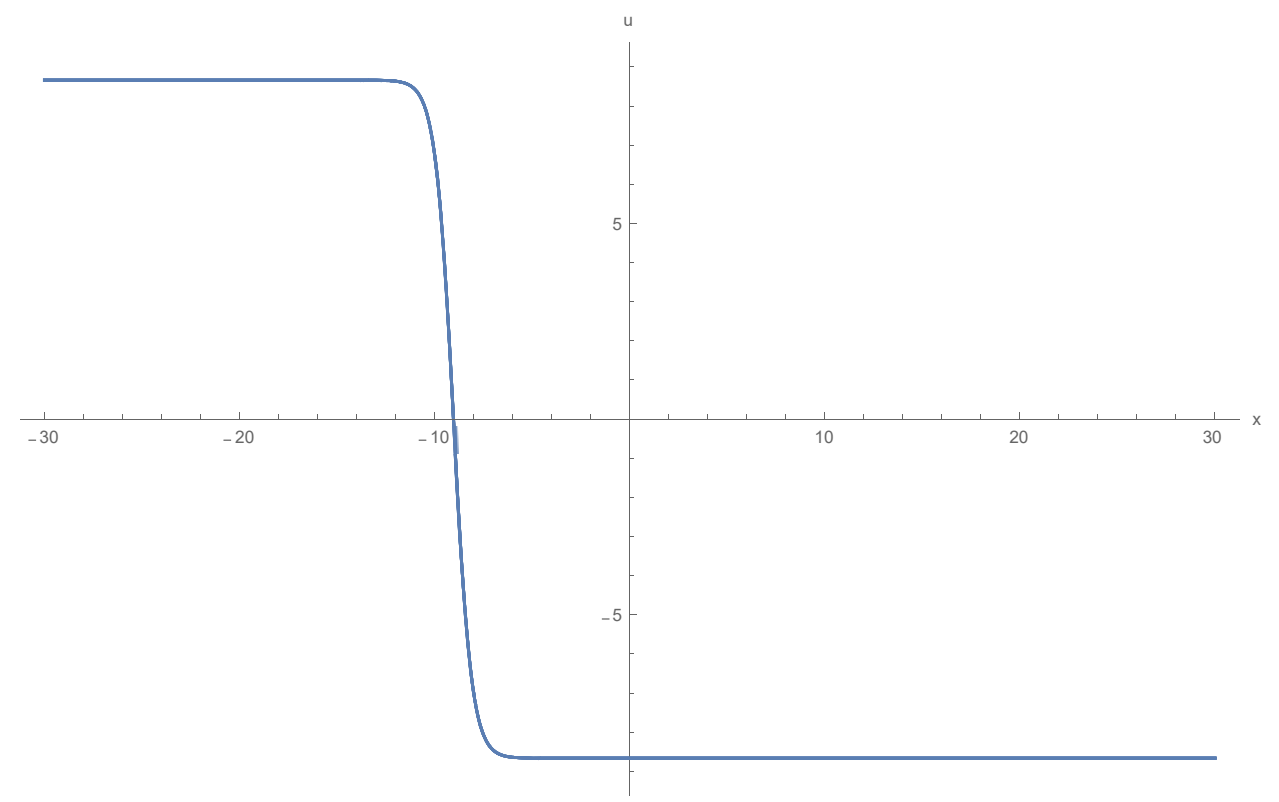

Figure 2: $\quad 2 \mathrm{D}$ plot of the obtained traveling wave solution $u_{4}(x, 0.25,1)$ of Eq.(21). 


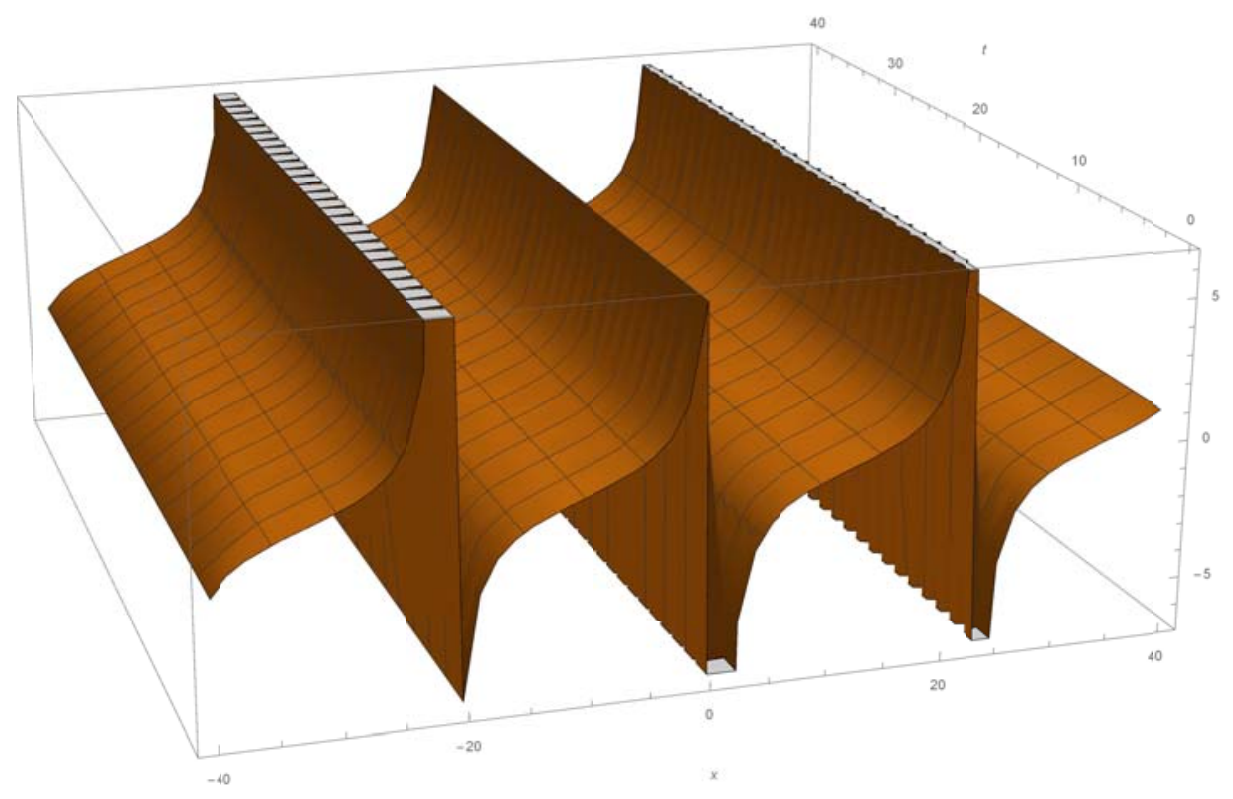

Figure 3: $3 \mathrm{D}$ plot of the obtained traveling wave solution $u_{8}(x, 1, t)$ of Eq.(23)

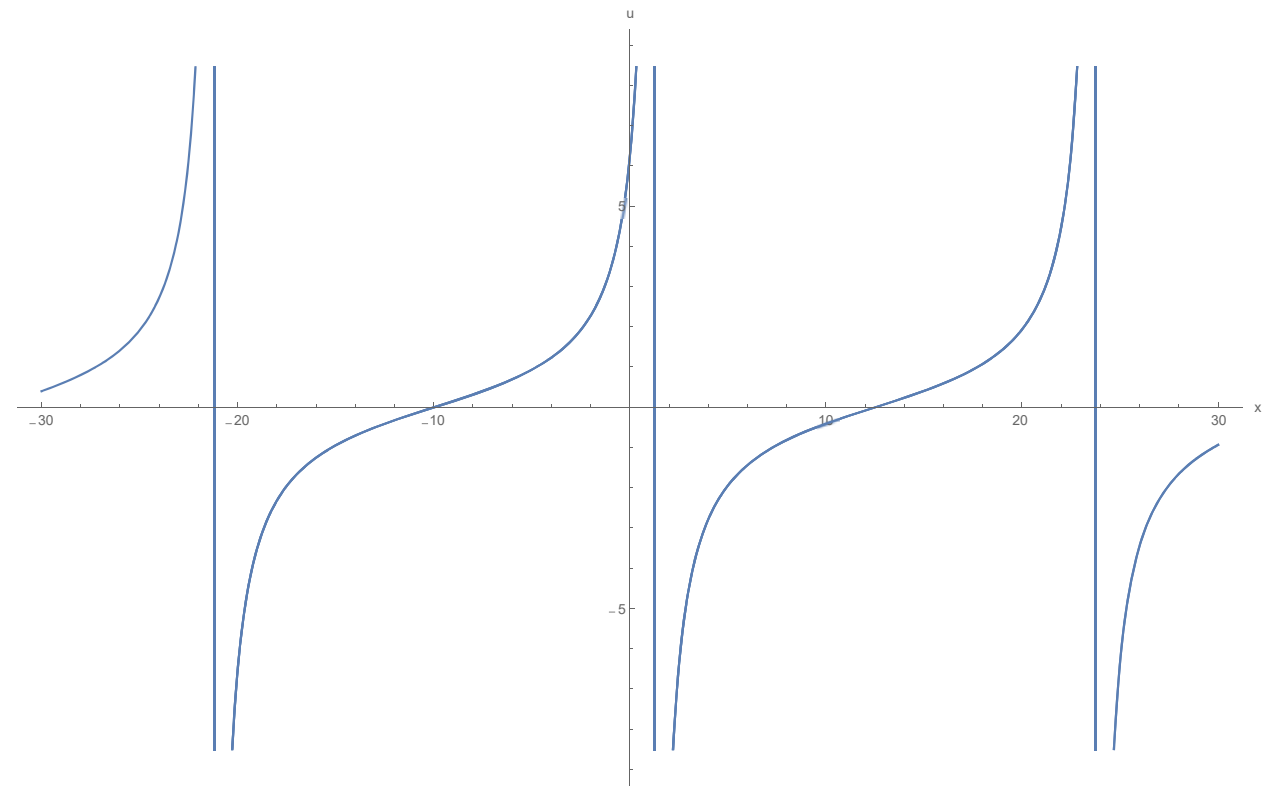

Figure 4: $2 \mathrm{D}$ plot of the obtained traveling wave solution $u_{8}(x, 1,1)$ of Eq.(23). 


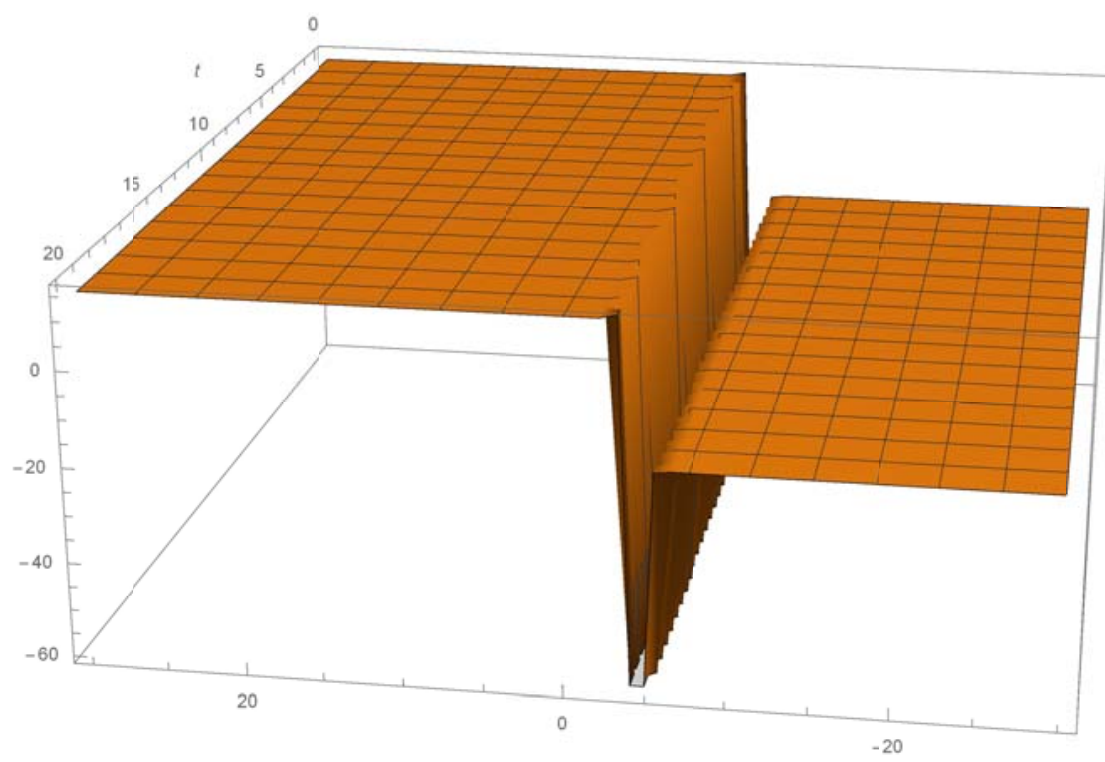

Figure 5: $3 \mathrm{D}$ plot of the obtained traveling wave solution $u_{12}(x, 1, t)$ of Eq.(25).

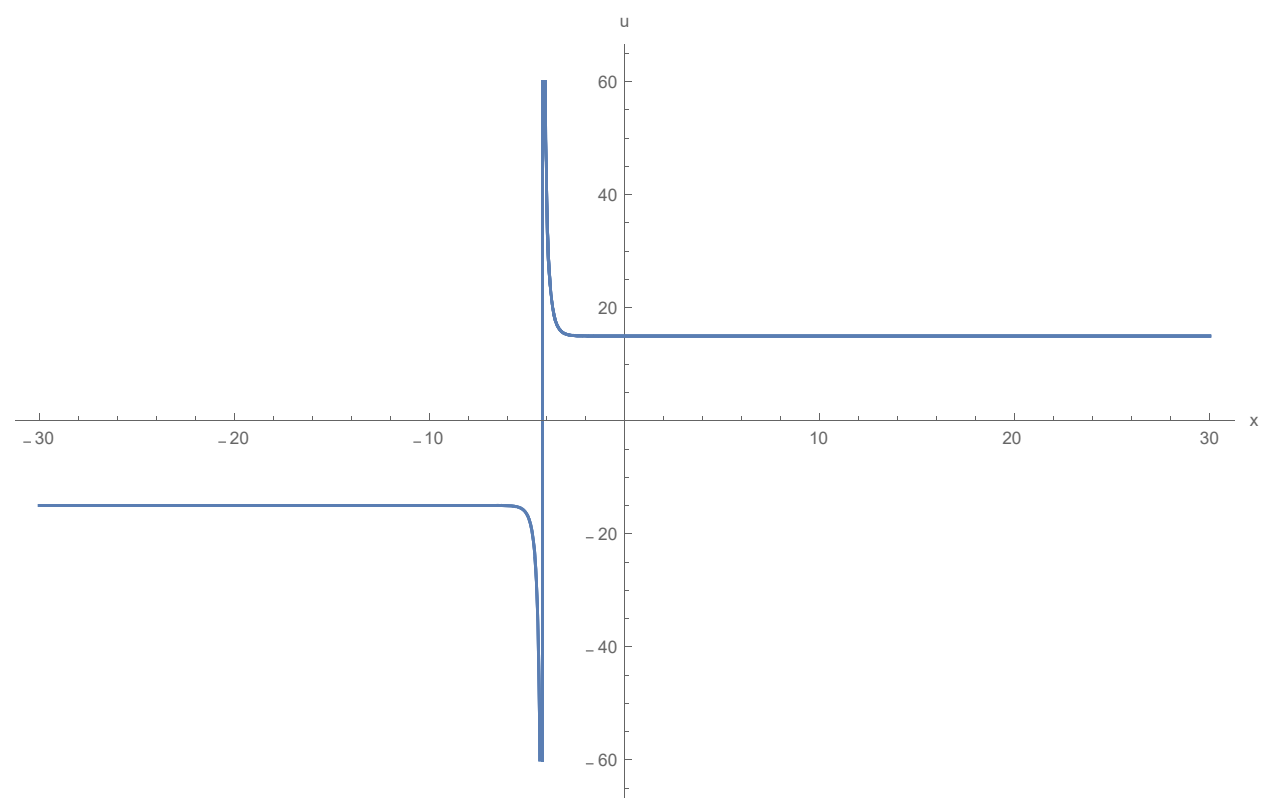

Figure 6: 2D plot of the obtained traveling wave solution $u(x, 1,1)$ of Eq.(25). 


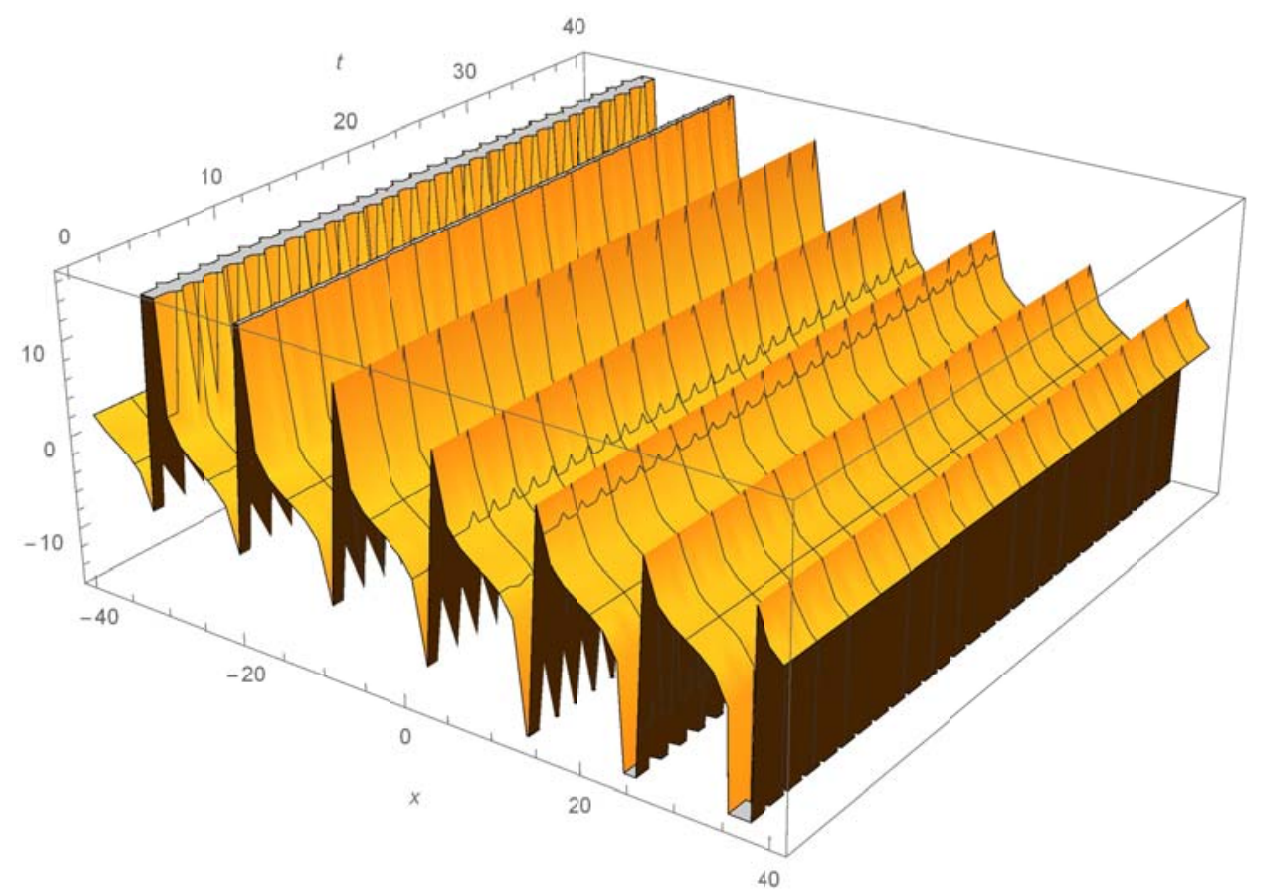

Figure 7: $\quad 3 \mathrm{D}$ plot of the obtained traveling wave solution $u_{18}(x, 1, t)$ of Eq.(28).

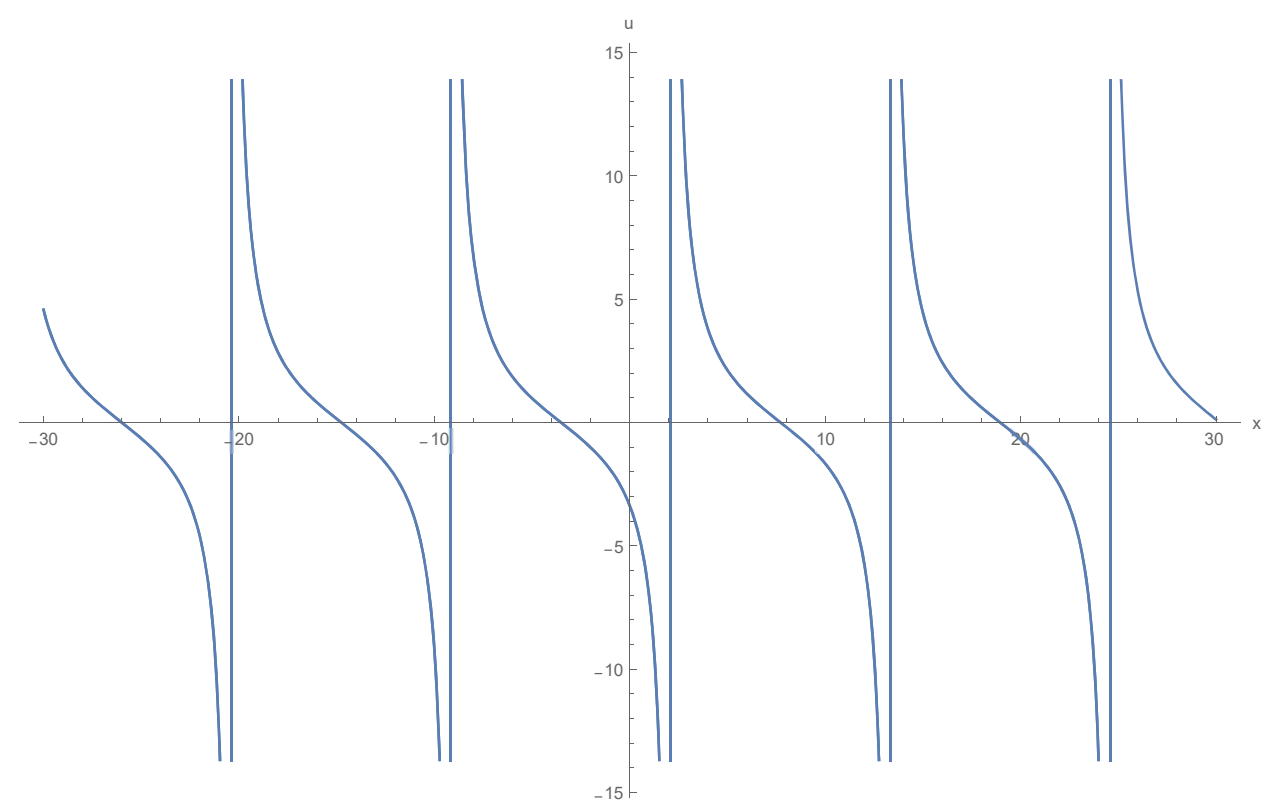

Figure 8: $\quad 2 \mathrm{D}$ plot of the obtained traveling wave solution $u_{18}(x, 1,1)$ of Eq.(28). 